\title{
Monte Carlo Methods for Adaptive Sparse Approximations of Time-Series
}

\author{
Thomas Blumensath and Michael E. Davies
}

(C)2007 IEEE. Personal use of this material is permitted. However, permission to reprint/republish this material for advertising or promotional purposes or for creating new collective works for resale or redistribution to servers or lists, or to reuse any copyrighted component of this work in other works must be obtained from the IEEE. 


\title{
Monte Carlo Methods for Adaptive Sparse Approximations of Time-Series
}

\author{
Thomas Blumensath, Member, IEEE, Mike E. Davies, Member, IEEE
}

\begin{abstract}
This paper deals with adaptive sparse approximations of time-series. The work is based on a Bayesian specification of the shift-invariant sparse coding model. To learn approximations for a particular class of signals, two different learning strategies are discussed. The first method uses a gradient optimisation technique commonly employed in sparse coding problems. The other method is novel in this context and is based on a sampling estimate. To approximate the gradient in the first approach we compare two Monte Carlo estimation techniques, Gibbs sampling and a novel importance sampling method. The second approach is based on a direct sample estimate and uses an extension of the Gibbs sampler used with the first approach. Both approaches allow the specification of different prior distributions and we here introduce a novel mixture prior based on a modified Rayleigh distribution.

Experiments demonstrate that all Gibbs sampler based methods show comparable performance. The importance sampler was found to work nearly as well as the Gibbs sampler on smaller problems in terms of estimating the model parameters, however, the method performed substantially worse on estimating the sparse coefficients. For large problems we found that the combination of a subset selection heuristic with the Gibbs sampling approaches can outperform previous suggested methods. In addition, the methods studied here are flexible and allow the incorporation of additional prior knowledge, such as the nonnegativity of the approximation coefficients, which was found to offer additional benefits where applicable.
\end{abstract}

Index Terms-Sparse approximation, time-series modelling, Monte Carlo approximation, importance and Gibbs sampling.

\section{INTRODUCTION}

Sparse approximations are signal approximations that use a linear combination of a small number of elementary waveforms selected from a large set of waveforms called a dictionary. The number of elements selected is in general much smaller than the dimension of the signal space and the size of the dictionary is often much larger. Such signal approximations have recently gained in popularity in the signal processing community with applications to source coding [1], [2], [3], machine learning [4], blind source separation [5], [6] and denoising [7].

For a general class of observations it is often not clear how to best select the dictionary to achieve the best trade-off between sparsity and reconstruction error. Several methods have therefore been proposed to adapt the dictionary for any given

The authors are with IDCOM \& Joint Research Institute for Signal and Image Processing, Edinburgh University, King's Buildings, Mayfield Road, Edinburgh EH9 3JL, UK (Tel.: +44(0)131 6505659, Fax.: +44(0)131 6506554, e-mail: thomas.blumensath@ed.ac.uk,mike.davies@ed.ac.uk).

This research was supported by EPSRC grant D000246/1. MED acknowledges support of his position from the Scottish Funding Council and their support of the Joint Research Institute with the Heriot-Watt University as a component part of the Edinburgh Research Partnership. set of observations [8] [9]. Optimal approximations exploit signal structures and automatic adaptation of the dictionary can lead to the discovery of such structures with the set of learned waveforms often representing salient signal features. Our focus in this papers is on applications in which it is desired to extract physically meaningful features from a signal. Many different signals encountered in engineering can be modelled with highly sparse approximations suggesting the use of sparse coding techniques to these problem domains. Recent examples can be found in biomedical applications [10] and [11] and in the analysis of musical signals [12], [13] and [14].

Learning algorithms developed to find optimally adapted sparse approximations are in general iterative [8] [9]. In most previous methods (e.g.[8], [9]) each iteration depends on estimates of the unknown sparse coefficients conditional on the data and the current estimate of the dictionary. Unfortunately, finding these approximations with the smallest number of nonzero coefficients and such that the approximation error is below a certain size is known to be an NP hard problem [15]. Instead, non-optimal strategies have been used such as greedy algorithms like matching pursuit and orthogonal matching pursuit [16] or convex (e.g. [17]) and non-convex (e.g. [18]) relaxations of the cost function. These have been used for adaptive sparse coding in, for example, [8], [9] and [19], whilst greedy methods have been used in [20] and [2].

A different approach, which does not rely on a single point estimate of the sparse coefficients in each iteration, is to use sampling based methods such as those suggested in [21], [22], [23] and [24]. These will be the starting point for the ideas presented in this paper.

One of the main drawbacks of the standard linear sparse approximation model for applications in signal processing is that the model assumes that the observations are vector valued. In signal processing one often deals not with vector valued data but with time-series. For such data, the structures and features in any observation can often occur at arbitrary locations and for optimal sparse approximations of such data, the dictionary has to be able to model structures at arbitrary shifts. Over the last few years, shift-invariant sparse coding formulations have therefore been introduced [25], [26], [27], [22], [28], [29] , [14], [13], [2] and [20].

In this paper we compare a range of learning algorithms based on Monte Carlo approximations to solve this shiftinvariant sparse coding problem. In particular, we propose a novel prior formulation, we propose a novel importance sampling algorithm and derive a novel Gibbs sampling method. For background on Gibbs and importance sampling, the reader should refer to standard textbooks such as [30].

We begin this paper with the specification of the shift- 
invariant sparse approximation model. The learning and inference problem in this model will be cast in a Bayesian framework allowing for the development of theoretical parameter learning rules in section III. The first rule is based on gradient optimisation. The evaluation of the gradient requires integration for which no analytical solutions are available and we suggest two Monte Carlo strategies in section IV to solve this problem. We use a Gibbs sampling approximation, which is described in subsection IV-A and derived an importance sampling approximation, which is presented in IV-B.

The other learning paradigm proposed is to directly sample from the parameter posterior and to use sample estimates of the parameters. To this end we extend the Gibbs sampler to also sample from the dictionary itself.

Section VI presents a comparison between the different Monte Carlo based approaches. We use a set of simplified test signals that allow us to study different properties of the algorithms. A slightly more difficult test signal is used in section VII to compare the Monte Carlo methods discussed here with several previously suggested methods.

\section{TheOreticAl BACKGROUND}

\section{A. The Shift-Invariant Linear Model}

The standard linear sparse approximation model introduced in [8] and [9] is often written as:

$$
\mathbf{x}=\mathbf{A s}+\epsilon
$$

where $\mathbf{x} \in \mathbb{R}^{M}$ is the observation vector we wish to approximate. $\mathbf{A} \in \mathbb{R}^{M \times N}$ is the dictionary matrix whose column vectors are the waveforms used to model $\mathbf{x}$. The vector $\mathbf{s} \in \mathbb{R}^{N}$ is the coefficient vector, which is assumed to be sparse, i.e. it is assumed to have only a small number of non-zero coefficients. The vector $\epsilon \in \mathbb{R}^{M}$ represents the approximation error. In this paper we assume that the error is i.i.d. Gaussian noise with scale parameter $\lambda_{\epsilon}$. We further assume an over-complete dictionary with $M<N$ so that there are infinitely many vectors $\mathbf{s}$ able to approximate $\mathbf{x}$ with the same approximation error. This model has also been studied as an over-complete and noisy version of Independent Component Analysis [31]. In this context, bayesian approaches have been proposed in [32] and in [33].

The location of characteristic features in time-series is often not known a priori and if the features can occur at arbitrary locations, then the matrix $\mathbf{A}$ has to include the same feature at different shifts, i.e. we are interested in finding a shift-invariant generative signal model. Such a model can be written as a mixture of convolutions [26]:

$$
x[t]=\sum_{k} \sum_{\tau} a_{k}[t-\tau] s_{k}[\tau]+\epsilon[t],
$$

where we now adopt a more familiar time-series notation. In this model the time-series of sparse coefficients $s_{k}[t]$ are convolved with the different features $\mathbf{a}_{k}$. We can also write this model in matrix notation as $\mathbf{x}=\mathbf{A} \mathbf{s}+\epsilon$, where $\mathbf{s}$ is now the vector containing the concatenation of the time series $s_{k}[t]$. The matrix $\mathbf{A}$ is then the concatenation of convolution matrices in which the features occur as column vectors, but repeated at different shifted locations. This structure is best understood from the following graphical representation:

$\left[\begin{array}{cccccccccccc}\star_{3} & \star_{2} & \star_{1} & 0 & 0 & 0 & \circ_{3} & \circ_{2} & \circ_{1} & 0 & 0 & 0 \\ 0 & \star_{3} & \star_{2} & \star_{1} & 0 & 0 & 0 & \circ_{3} & \circ_{2} & \circ_{1} & 0 & 0 \\ 0 & 0 & \star_{3} & \star_{2} & \star_{1} & 0 & 0 & 0 & \circ_{3} & \circ_{2} & \circ_{1} & 0 \\ 0 & 0 & 0 & \star_{3} & \star_{2} & \star_{1} & 0 & 0 & 0 & \circ_{3} & \circ_{2} & \circ_{1}\end{array}\right]$

We here show $\mathbf{A}$ for two features, represented with stars $\star$ and circles $\circ$ respectively. The subscripts label the individual samples in each feature. In the following we will use the notation A exclusively to refer to this structured matrix. For infinite time-series, the length of the vectors $\mathbf{x}, \mathbf{s}$ and $\epsilon$ as well as the dimension of the matrix $\mathbf{A}$ would be infinite. When dealing with long time-series, it is customary to deal with the data in blocks, which we also model as $\mathbf{x}=\mathbf{A s}+\epsilon$ by shrinking the matrix $\mathbf{A}$ and the vector $\mathbf{s}$ accordingly ${ }^{1}$.

\section{B. Sparse Approximations}

If the features $\mathbf{a}_{k}$ are known, we are still left with the problem of finding $\mathbf{s}$ for any given $\mathbf{x}$. The problem of sparse approximations is often stated in terms of different optimisation problems. For example we could try to find a representation $\mathbf{s}$ with as few non-zero coefficients as possible such that the norm of the error $\epsilon$ is below some value or we could try to minimise the reconstruction error subject to the number of non-zero coefficients being below some value. Both of these problems can be expressed using the Lagrangian form:

$$
\arg \min _{s}\|\mathbf{x}-\mathbf{A s}\|_{2}^{2}+\lambda\|\mathbf{s}\|_{0}
$$

for some $\lambda$. Here $\|\cdot\|_{0}$ is the numerosity or the number of non-zero coefficients in $\mathbf{s}$.

Unfortunately, the numerosity is not an easy function to deal with in the above optimisation setting. A standard method to approximate the numerosity is to use any of the $L_{p}$ quasi norms with $0<p \leq 1$, which leads to the optimisation problem:

$$
\arg \min _{s}\|\mathbf{x}-\mathbf{A} \mathbf{s}\|_{2}^{2}+\lambda\|\mathbf{s}\|_{p}^{p}
$$

\section{Bayesian interpretation}

Bayesian methods take a different view from the optimisation based approach based on equation (4). It is true, equation (4) can be derived as a maximum a posterior (MAP) estimate for certain probabilistic models ${ }^{2}$, however, the Bayesian approach does not start with the specification of an objective function such as equation (4), instead one specifies a probabilistic model, from which, at least in theory, marginal and posterior distributions can be evaluated. An objective function is then specified for parameters of interest, with the choice of the measure depending on the application studied.

\footnotetext{
${ }^{1}$ Note that one could also specify a shift-invariant model in the frequency domain by discarding phase information. The model used here differs from these methods in that the features $\mathbf{a}_{k}$ also model the phase. The differences between phase blind methods and the model discussed here has been studied in detail in [14].

${ }^{2}$ For example in [34] the Laplacian distribution was suggested as a prior for $p\left(s_{n}\right)$.
} 
The models used in this paper are based on the factorial mixture prior on the coefficients s suggested in [35] and [22]:

$$
p(\mathbf{s} \mid \mathbf{u})=\prod_{n} p\left(s_{n} \mid u_{n}\right)=\prod_{n}\left(u_{n} p\left(s_{n}\right)+\left(1-u_{n}\right) \delta_{0}\left(s_{n}\right)\right),
$$

where $u_{n} \in\{0,1\}$ is a binary indicator variable specifying whether $s_{n}$ is zero or non-zero, with discrete distribution ${ }^{3}$ :

$$
p\left(u_{n}\right)=\theta^{u_{n}}+(1-\theta)^{\left(1-u_{n}\right)}=\frac{1}{1+e^{-\frac{\lambda u}{2}}} e^{-\frac{\lambda u}{2} u_{n}},
$$

$\delta_{0}\left(s_{n}\right)$ is the Dirac mass at zero and $p\left(s_{n}\right)$ is the density of the non-zero $s_{n}$. Note that in [35] and [22] the prior for the nonzero coefficients was assumed to be i.i.d. Gaussian, however, a range of priors can be used, some of which will be discussed in more detail in III-B.

\section{Monte Carlo Methods for Sparse Approximations}

Monte Carlo methods are statistical sampling based approximation procedures to evaluate statistical expectations [30]. These methods often rely on a set of samples drawn with a Markov chain sampler, such as the Metropolis-Hastings algorithm or the Gibbs sampler. In these methods, the expectations are evaluated by calculating the sample mean of the functions of interest evaluated at the sample points. Importance sampling [30] is a variant in which samples are drawn from another distribution while the expectations with respect to the target distribution are estimated using a weighted sample mean.

If a probabilistic model is used for the sparse coefficients in a sparse approximation model, it is possible to develop a Markov chain sampler to draw samples from this distribution. In such a setting, the Markov chain sampler can be understood as a stochastic search procedure that explores the coefficient distribution.

The performance of a Gibbs sampler strongly depends on the ability of the sampler to explore the distribution. For sparse models, the coefficient distribution is in general multimodal [36]. Sampling from such a distribution is difficult and a very large number of samples are often required to ensure that the sampler explores the full distribution. For highly multimodal distributions (such as those observed in the experiments in this paper), the chain can often get stuck around certain modes. In many practical applications one is often only able to draw a small number of samples and it can not be assumed that the samples represent the full distribution. The exact part of the distribution that is explored then depends strongly on the first sample in the chain, which leads to a biased estimate (see below) of the expectation to be evaluated.

The performance of an importance sampler depends on the used proposal distribution, which influences the variance of the estimate. In this paper we use an importance sampler to estimate a gradient in a stochastic gradient descent procedure. As the variation in the gradient estimate is averaged out over many iterations [37] we found that the increase in variance when using the importance sampler instead of the Gibbs sampler did not pose any problems in the optimisation method.

\footnotetext{
${ }^{3}$ Note that the non-standard form of the Bernoulli distribution with parameter $\lambda_{u}$ given on the right is introduced to simplify the notation.
}

More critical is the bias introduced by normalisation of the sample weights when the distributions are only available up to a normalising constant. To better understand this problem assume we want to optimise a function $g(\mathbf{s})$. A fixed point is found where the gradient $\Delta g(\mathbf{s})=0$. However, instead of the gradient $\Delta g(\mathbf{s})$ we only have an estimate of this gradient, say $\widehat{\Delta g(\mathbf{s})}$. A stochastic gradient procedure requires that the expectation of the gradient estimate is unbiased [37], i.e that $\Delta g(\mathbf{s})=E\{\widehat{\Delta g(\mathbf{s})}\}$.

Sampling methods enable us to calculate different estimates of the sparse coefficients. In Bayesian statistics one often takes the mean as an estimate as this would minimise a squared error loss function. However, for sparse approximations this approach is questionable. The sample mean is in general less sparse than any of the individual samples. For this reason, the maximum a posteriori (MAP) value seems to be a better estimate. A MAP estimate can be found from the samples drawn with any sampling strategy by choosing the samples for which this distribution is maximal. Another method is to use annealing techniques. However, we found that an annealing method did not offer any significant advantages.

\section{DICTIONARY LEARNING}

In many applications we want to learn the features $\mathbf{a}_{k}$ to best model a set of observations. The statistical framework introduced above allows us to develop algorithms to adapt the features $\mathbf{a}_{k}$ for any particular set of signals. In the standard linear model, adaptive sparse approximations were developed in [8] and [34]. The learning rule in [8] can be adapted to the shift-invariant model as in [26]. From a Bayesian point of view, an optimal estimate for $\mathbf{A}$ would be the mean estimate (under a squared error loss) or the MAP estimate (under a 'zero-one' loss) of $p(\mathbf{A} \mid \mathbf{x})$. Estimation strategies for these two estimates are discussed in subsections III-B and III-A respectively. The mean estimation procedure proposed in subsection III-B below is based on a sample estimate of the mean of $p(\mathbf{A} \mid \mathbf{x})$ calculated from samples drawn with a Markov chain. The features $\mathbf{a}_{k}$ do not have any natural ordering and any permutation of the indices $k$ in the model will lead to a posterior with the same probability. Calculating the mean of such a distribution will in theory result in features $\mathbf{a}_{k}$ that are all equal ${ }^{4}$. We found that this problem is avoided in practice by the fact that the Markov chain does not fully explore the posterior distribution and in general mainly samples from a single mode of the posterior. However, it has to be recognised that the reported results are not due to a full Bayesian analysis but due to the shortcomings of the chain to explore the full posterior distribution. For a full Bayesian analysis other methods have been proposed in [38] and [39] to solve the inference problem in mixture distributions. These include clustering of the posterior samples, the use of ordering constraints and the optimisation of permutation invariant utility functions.

\footnotetext{
${ }^{4}$ A simple example would be a mixture of two univariate Guassians where the Gaussians only vary in their mean. The mean of such a mixture distribution lies exactly between the means of the individual Gaussians [38].
} 
The MAP estimation method discussed in subsection IIIA uses a gradient based method to find a maximum of the posterior $p(\mathbf{A} \mid \mathbf{x})$ and will converge to a single mode of the distribution. This approach therefore does not suffer from the same problem.

\section{A. Learning Strategy 1: Gradient Based Learning}

In the first method we calculate a MAP estimate of the marginalised posterior:

$$
p(\mathbf{A} \mid \mathbf{x}) \propto p(\mathbf{A}) \int p(\mathbf{x} \mid \mathbf{A}, \mathbf{s}) p(\mathbf{s}) d \mathbf{s} .
$$

The integration in equation (7) cannot be solved analytically and no direct maximum a posteriori estimate is available. Instead, we derive a gradient based optimisation method by writing the gradient of the logarithm of the marginalised posterior using the derivation given in appendix I:

$$
\Delta \mathbf{a}_{k}=\frac{\partial \log p(\mathbf{A} \mid \mathbf{x})}{\partial \mathbf{a}_{k}}=\left\langle\frac{\partial}{\partial \mathbf{a}_{k}} \log p(\mathbf{x}, \mathbf{A} \mid \mathbf{s})\right\rangle_{p(\mathbf{s} \mid \mathbf{A}, \mathbf{x})} .
$$

There is a scale ambiguity between the norm of the features $\mathbf{a}_{k}$ and the coefficients $\mathbf{s}$, so that we normalise the features $\mathbf{a}_{k}$ to unit $L_{2}$ norm after each update. The features $\mathbf{a}_{k}$ are therefore updated using the gradient step:

$$
\mathbf{a}_{k}^{r+1}=\frac{\mathbf{a}_{k}^{r}+\nu \Delta \mathbf{a}_{k}}{\left\|\mathbf{a}_{k}^{r}+\nu \Delta \mathbf{a}_{k}\right\|_{2}},
$$

where $\nu$ is a learning rate and $r$ the iteration counter.

The expectation in equation (8) cannot be evaluated analytically, however, different methods can be used to approximate this gradient. In [40] different analytical gradient approximations have been proposed for the standard sparse approximation problem. We discuss two approximations based on Monte Carlo methods. The first method (proposed in [22] with a Gaussian prior for $\left.p\left(s_{n} \mid u_{n}\right)\right)$ is based on a Gibbs sampler while the second approach is novel and uses the weighted samples drawn with an importance sampling method.

Due to the assumed i.i.d. Gaussian noise model we have $p(\mathbf{x} \mid \mathbf{A}, \mathbf{s}) \sim \mathcal{N}\left(\mathbf{A s}, \lambda_{\epsilon}^{-1}\right)$. Assuming a flat prior for $p(\mathbf{A})$ we get a gradient of:

$$
\Delta a_{k}[m]=\sum_{j=1}^{J} w_{j} \sum_{t} \epsilon[t] \hat{s}_{k, j}[m-t],
$$

where $\epsilon[t]=x[t]-\sum_{k} \sum_{\tau} a_{k}[t-\tau] \hat{s}_{k, j}[\tau]$. The subscript $j$ in the notation $\hat{s}_{k, j}$ refers to the $j^{\text {th }}$ sample drawn from $p(\mathbf{s} \mid \mathbf{x}, \mathbf{A})$ and $J$ is the total number of samples drawn. We here introduce the importance weights $w_{j}$ associated with the importance estimation procedure [30], when using the Gibbs sampler, the weights $w_{j}$ in equation (10) are set to $\frac{1}{J}$.

Estimates calculated with Monte Carlo methods are stochastic and therefore lead to a stochastic gradient descent optimisation procedures [37]. With such an approach there exists a trade-off between the number of overall iterations and the accuracy with which each individual gradient is estimated. This is the motivation behind the importance sampling approximation, which can be used to calculate a fast, but more noisy estimate of the gradient in each iteration. Furthermore, the stochastic gradient formulation naturally allows us to use just a small block $\mathbf{x}$ taken at a random location from the timeseries in each iteration. The full data set does therefore not have to be kept in memory and the method is well suited for applications in which new data becomes available sequentially.

If dealing with blocks instead of the complete time-series, end-effects have to be taken into account. For example, when inferring $\mathbf{s}$ for a given observation block $\mathbf{x}$ and a model matrix A, less information is available in the observation for those coefficients $\mathbf{s}$ for which the associated column in $\mathbf{A}$ only contains a small part of a feature $\mathbf{a}_{k}$ [13]. The advantage of the Monte Carlo methods studied in this paper is that this uncertainty is reflected in the full posterior distribution $p(\mathbf{s} \mid \mathbf{x}, \mathbf{A})$, so that the heuristics suggested with previous approaches [26] and [13] are not required.

\section{B. Learning Strategy 2: Sampling Based Learning}

The second possible approach is to estimate $\mathbf{a}_{k}$ using the sample mean of samples drawn from $p(\mathbf{A} \mid \mathbf{x})$. Such an approach is similar in spirit to the method used in [33] for blind source separation, however, the method proposed here is different, both in terms of the prior model and the structure and size of $\mathbf{A}$.

A block Gibbs sampler can be used to jointly sample from $p(\mathbf{A}, \mathbf{s} \mid \mathbf{x})$ by alternatively sampling from the conditional distributions $p(\mathbf{A} \mid \mathbf{s}, \mathbf{x})$ and $p(\mathbf{s} \mid \mathbf{A}, \mathbf{x})$. A method to sample from $p(\mathbf{s} \mid \mathbf{A}, \mathbf{x})$ is described in the next section, whilst samples from $p(\mathbf{A} \mid \mathbf{s}, \mathbf{x})$ can be drawn by sequentially sampling from $p\left(\mathbf{a}_{k} \mid\left\{\mathbf{a}_{n \neq k}\right\}, \mathbf{s}, \mathbf{x}\right)$ for all $k$. Here $\left\{\mathbf{a}_{n \neq k}\right\}$ is the set of features excluding the $k^{t h}$ feature.

With the assumption of an i.i.d. Gaussian prior with scale parameter $\lambda_{a}$ on the elements of $\mathbf{a}_{k}$ we can write the posterior $p\left(\mathbf{a}_{k} \mid\left\{\mathbf{a}_{n \neq k}\right\}, \mathbf{s}, \mathbf{x}\right)$ as

$$
p\left(\mathbf{a}_{k} \mid\left\{\mathbf{a}_{n \neq k}\right\}, \mathbf{s}, \mathbf{x}\right) \sim \mathcal{N}\left(\mu_{k}, \Sigma_{k}\right),
$$

where we have

$$
\Sigma_{k}=\left(\lambda_{\epsilon}\left(\mathbf{S}_{k}^{T} \mathbf{S}_{k}\right)+\lambda_{a} \mathbf{I}\right)^{-1}
$$

and

$$
\mu_{k}=\lambda_{\epsilon} \Sigma_{k} \mathbf{S}_{k}^{T} \epsilon_{k} .
$$

We here use $\epsilon_{k}=\mathbf{x}-\sum_{n \neq k} \sum_{\tau} a_{n}[t-\tau] \hat{s}_{n}[\tau]$, where $\hat{s}_{n}[\tau]$ are the current samples from $p(\mathbf{s} \mid \mathbf{A x})$. We further define $\mathbf{S}_{k}$ by the relationship:

$$
\sum_{l} a_{k}[t-\tau] s_{k}[\tau]=\mathbf{S}_{k} \mathbf{a}_{k}
$$

$\mathbf{S}_{k}$ is therefore a convolution matrix similar to the definition of A but this time containing the time series $s_{k}[t]$, i.e. we express the convolution of a single feature with the associated time series using matrix notation. We therefore have the equivalent matrix formulations for the model:

$$
\mathbf{A s}=\left[\mathbf{S}_{1} \mathbf{S}_{2} \cdots \mathbf{S}_{K}\right]\left[\begin{array}{c}
\mathbf{a}_{1} \\
\mathbf{a}_{2} \\
\vdots \\
\mathbf{a}_{K}
\end{array}\right]
$$


Note that we fix the variance $\lambda_{a}$ a priori and we found that this constrains the algorithm sufficiently so that we do not have to re-normalise the features $\mathbf{a}_{k}$.

In contrast to the stochastic gradient methods of the previous section, this formulation is intrinsically based on all available data $\mathrm{x}$.

\section{SAMPling BASEd SPARSE APPROXIMATIONS}

Both learning strategies discussed above require samples to be drawn from $p(\mathbf{s} \mid \mathbf{x}, \mathbf{A})$. This can be done by drawing samples from the joint distribution $p(\mathbf{s}, \mathbf{u} \mid \mathbf{x}, \mathbf{A})$ and then dropping the samples $\hat{\mathbf{u}}$. In the first part of this section we discuss different Gibbs sampling strategies to draw samples from the mixture distribution. For the gradient based learning strategy another possibility is to replace samples drawn from $p(\mathbf{s}, \mathbf{u} \mid \mathbf{x}, \mathbf{A})$ with samples drawn from any other distribution with the same support and to use importance sampling to estimate the gradient. Such an approach is discussed in subsection IV-B.

\section{A. Sampling Method 1: Gibbs Sampling}

In [21] and [22] two Gibbs sampling algorithms were proposed to solve the problem of learning an over-complete dictionary matrix $\mathbf{A}$ for sparse signal approximations. Similar sampling methods were previously suggested in [35], [41], [42], [43], [44], [45], [46] for the subset selection problem in regression. These methods are based on a mixture prior similar to the one used in this paper and a range of different distributions for the non-zero coefficients can be used, some of which are discussed below.

For the mixture model discussed here, different implementations are possible in order to draw samples from $\mathbf{u}$ and $\mathbf{s}$. For a mixture of Gaussians it is possible to draw samples $p\left(u_{n} \mid\left\{u_{\hat{n} \neq n}\right\}, \mathbf{s}, \mathbf{x}, \mathbf{A}\right)$ and $p\left(\mathbf{s}_{n} \mid\left\{s_{\hat{n} \neq n}\right\}, \mathbf{u}, \mathbf{x}, \mathbf{A}\right)$ [47], i.e. by standard Gibbs sampling from the conditional densities, where the subscript notation $\hat{n} \neq n$ refers to quantities with subscripts other than $n$. The problem with this method is that for mixtures of Gaussians, in which each Gaussian has a very different variance, the chain seldom switches states [35]. An extreme case would be the mixture of a Gaussian and a delta function, in which, whenever $s_{n}$ is non-zero, the chain is not able to change the variable $u_{n}$, as such a change would have zero probability. In order to overcome this problem it is possible to sample from $p\left(u_{n} \mid\left\{u_{\hat{n} \neq n}\right\}, \mathbf{x}, \mathbf{A}\right)$ [35], [21], i.e. by integrating out the coefficients $\mathrm{s}$.

However, the evaluation of $p\left(u_{n} \mid\left\{u_{\hat{n} \neq n}\right\}, \mathbf{x}, \mathbf{A}\right)$ is computationally demanding. This can be avoided by only integrating out a single coefficient $s_{n}$, i.e by sampling from

$$
p\left(u_{n} \mid\left\{s_{\hat{n} \neq n}\right\},\left\{u_{\hat{n} \neq n}\right\}, \mathbf{x}, \mathbf{A}\right) .
$$

After sampling of the indicator variable from $p\left(u_{n} \mid\left\{s_{\hat{n} \neq n}\right\},\left\{u_{\hat{n} \neq n}\right\}, \mathbf{x}, \mathbf{A}\right)$ it is then easy to sample from $p\left(s_{n} \mid\left\{\mathbf{s}_{\hat{n} \neq n}\right\}, \mathbf{u}, \mathbf{x}, \mathbf{A}\right)$. In effect, this method samples directly form the joint distribution

$p\left(s_{n}, u_{n} \mid\left\{s_{\hat{n} \neq n}\right\},\left\{u_{\hat{n} \neq n}\right\}, \mathbf{x}, \mathbf{A}\right)$. This is the approach adopted here. It is worth pointing out that in general it is desirable to sample from larger blocks of parameters at once to improve Markov chain mixing. However, for the application of interest here, the cost involved in the required matrix calculations was found to outweigh the mixing advantage.

In order to use the Gibbs strategy, it is beneficial to choose a prior distribution that facilitates the integration over a single coefficient $s_{n}$. In general it is possible to use any mixture model in which any of the distributions $p\left(s_{n} \mid u_{n}=1\right)$ and $p\left(s_{n} \mid u_{n}=0\right)$ have a Gaussian, delta, uniform, Rayleigh or modified Rayleigh distribution ${ }^{5}$. Other non-negative distributions for the non-zero coefficients are possible and the distributions above can also be used if they are restricted to positive values.

If we integrate out a single coefficient $s_{n}$ we have:

$$
\begin{aligned}
p\left(u_{n}=1 \mid\left\{s_{\hat{n} \neq n}\right\}, \mathbf{x}, \mathbf{A}\right) & =\frac{p\left(u_{n}=1 \mid\left\{s_{\hat{n} \neq n}\right\}, \mathbf{x}, \mathbf{A}\right)}{\sum_{k=0}^{1} p\left(u_{n}=k \mid\left\{s_{\hat{n} \neq n}\right\}, \mathbf{x}, \mathbf{A}\right)} \\
& =\frac{1}{1+e^{-E_{1}}},
\end{aligned}
$$

where

$$
E_{1}=\log \frac{p\left(u_{n}=1 \mid\left\{s_{\hat{n} \neq n}\right\}, \mathbf{x}, \mathbf{A}\right)}{p\left(u_{n}=0 \mid\left\{s_{\hat{n} \neq n}\right\}, \mathbf{x}, \mathbf{A}\right)} .
$$

The conditional distributions have therefore only to be known up to a normalising constant.

For different distributions of $p\left(s_{n} \mid u_{n}=1\right)$, different expressions for $E_{1}$ are found. In appendix III we derive the expression for two cases, the case in which $p\left(s_{n} \mid u_{n}=1\right)$ is the modified Rayleigh distribution discussed in appendix II and the case in which $p\left(s_{n} \mid u_{n}=1\right)$ is a normal distribution. Expressions for any of the other mentioned distributions and models follow similar derivations.

Conditional on $u_{n}$ it is then possible to draw samples from $p\left(s_{n} \mid\left\{s_{\hat{n} \neq n}\right\}, u_{n}=1, \mathbf{A}\right) \sim p\left(s_{n} ; \eta_{n}, \Psi_{n}^{-1}\right)$. For a Gaussian prior on the non-zero coefficients, this conditional posterior is also Gaussian with mean $\eta_{n}$ and variance $\Psi_{n}^{-1}$ defined by equation (27) and equation (28) in appendix III. The modified Rayleigh distribution is also a conjugate prior for the Gaussian mean and the conditional posterior therefore also a modified Rayleigh distribution with exactly the same parameters $\eta_{n}$ and $\Psi_{n}^{-1}$ as used in the Gaussian case. A method to draw samples from the modified Rayleigh distribution is presented in appendix IV.

\section{B. Sampling Method 2: Importance Sampling}

To evaluate the gradient in equation (8) used in the MAP estimation method in subsection III-A we propose an alternative Monte Carlo method based on importance sampling.

If we draw samples from a proposal distribution $q(\mathbf{s}, \mathbf{u})$ with the same support as $p(\mathbf{s}, \mathbf{u} \mid \mathbf{x}, \mathbf{A})$ and if we calculate the weights in equation (10) as

$$
w_{j}=\frac{1}{J} \frac{p\left(\hat{\mathbf{s}}_{j}, \hat{\mathbf{u}}_{j} \mid \mathbf{x}, \mathbf{A}\right)}{q\left(\hat{\mathbf{s}}_{j}, \hat{\mathbf{u}}_{j}\right)}=\frac{1}{J} \frac{p\left(\hat{\mathbf{s}}_{j} \mid \hat{\mathbf{u}}_{j}, \mathbf{x}, \mathbf{A}\right) p\left(\hat{\mathbf{u}}_{j} \mid \mathbf{x}, \mathbf{A}\right)}{q\left(\hat{\mathbf{s}}_{j}, \hat{\mathbf{u}}_{j}\right)},
$$

then the gradient estimate will be unbiased. Unfortunately, some of the distributions in the above calculation are only

\footnotetext{
${ }^{5}$ This distribution is defined in appendix II. It is a conjugate prior for the Gaussian mean and to our knowledge has not been used before.
} 
known up to a normalisation constant so that the weights have to be normalised, which, as discussed in II-D, does introduce a bias in the importance sampling estimate.

The bias and variance of the importance sampling estimate depend on the used proposal distribution, which should be close to the distribution of interest. We therefore propose a data dependent proposal distribution of the form $q(\mathbf{s}, \mathbf{u})=$ $p(\mathbf{s} \mid \mathbf{u}, \mathbf{x}, \mathbf{A}) \alpha(\mathbf{u} \mid \mathbf{x})$. Here $p(\mathbf{s} \mid \mathbf{u}, \mathbf{x}, \mathbf{A})$ is the true conditional posterior distribution which we assume can be sampled from directly, while for $\alpha(\mathbf{u} \mid \mathbf{x})=\prod_{n} \alpha\left(u_{n} \mid \mathbf{x}\right)$ we use the heuristic proposal distribution $\alpha\left(u_{n}=1 \mid \mathbf{x}\right)=p\left(u_{n}=1\right) * f_{n}(\mathbf{x})$, with

$$
f_{n}(\mathbf{x})=2 * \frac{\left|[\mathbf{A}]_{n}^{T} \mathbf{x}\right|^{0.4}}{\max _{\hat{n}}\left|[\mathbf{A}]_{\hat{n}}^{T} \mathbf{x}\right|},
$$

where $[\mathbf{A}]_{n}$ is the $n^{t h}$ column of $\mathbf{A}$. The non-linearity $f_{n}(\mathbf{x})$ given above has been chosen empirically to give a small variance in the weights for the experiments reported below.

\section{Summary of Computational Strategies}

We can now combine the two learning strategies of section III with the two sampling methods of section IV in the following ways:

- Grad + Gibbs: Gradient learning using the Gibbs sampler to evaluate the gradient;

- Grad + IS: Gradient learning using the importance sampler to evaluate the gradient;

- full Gibbs: Sample mean estimation of A using samples drawn from $p(\mathbf{A}, \mathbf{s} \mid \mathbf{x})$ by alternatively sampling from $p(\mathbf{s} \mid \mathbf{x}, \mathbf{A})$ and $p(\mathbf{A} \mid \mathbf{s}, \mathbf{x})$.

Each of the methods can be implemented using a range of different prior distributions for the non-zero coefficients.

\section{PERformance AnAlysis}

In this subsection we analyse the different Monte Carlo strategies and two different prior distributions on a simplified test signal and study the performance when changing certain parameters of the analysed signal.

\section{A. Test Signal}

The five features used to construct all test signals are shown in figure 1 . These features were chosen as the maximum correlation between different features is low, but the periodicity of the features means that they are strongly correlated with shifts of themselves. We generated four different test signals by drawing the non-zero coefficients from the modified Rayleigh distribution. The used parameters are listed in table I. The test signals were generated so that the first signal can be used as a reference, with the other signals varying one parameter relative to the first signal (i.e. increased noise (2), reduced sparsity (3) and reduced occurrence of feature $\left.a_{5}(4)\right)$.

\section{B. Estimation of $\mathbf{A}$}

In applications in which the features represent physically meaningful signal structures, it is often important to compute accurate estimates of these features. We therefore evaluate the performance of the methods in terms of estimates of $\mathbf{A}$. We

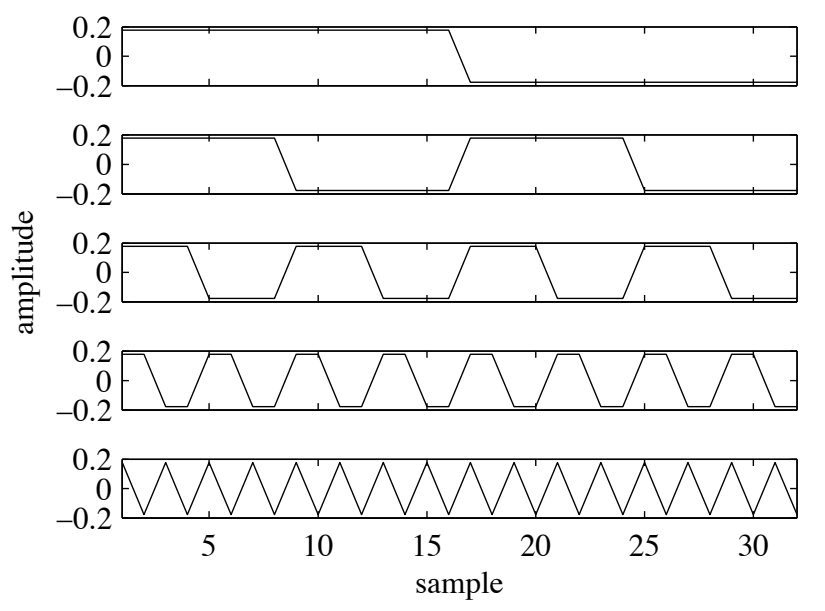

Fig. 1. Features used to generate the test signal.

TABLE I

TEST SIGNAL PARAMETERS.

\begin{tabular}{|l|c|c|c|c|}
\hline & 1 & 2 & 3 & 4 \\
\hline$\lambda_{\epsilon}\left(\sigma_{\epsilon}^{2}\right)$ & $100(0.01)$ & $10(0.1)$ & $100(0.01)$ & $100(0.01)$ \\
$\lambda_{u}(p(u=1))$ & $9(0.011)$ & $9(0.011)$ & $7.5(0.023)$ & $9(0.011)$ \\
Feature likelihood & equal & equal & equal & unequal \\
SNR & 13 & 2.7 & 15.8 & 13 \\
\hline
\end{tabular}

here assumed that all parameters were known to the algorithm apart from the true features and sparse coefficients ${ }^{6}$.

1) Used Algorithms: We run the following four algorithms on all four data sets:

- full NN Gibbs: The algorithm that samples from A and $\mathbf{s}$ with the modified Rayleigh prior on the non-zero coefficients and uses the sample mean to estimate $\mathbf{A}$;

- Grad + NN Gibbs: The gradient algorithm in which the gradient is estimated using Gibbs sampling based on the non-negative modified Rayleigh prior;

- Grad + Gauss Gibbs: The gradient algorithm in which the gradient is estimated using Gibbs sampling based on the Gaussian prior as previously suggested in [22];

- Grad + IS: The gradient algorithm in which the gradient is estimated using importance sampling based on the Gaussian prior.

Note that we here vary the used algorithms and the used models individually, allowing a direct comparison between the algorithmic approaches and the models, e.g. methods (full NN Gibbs) and (Grad + NN Gibbs) only differ in the used algorithms, while methods (Grad + Gauss Gibbs) and (Grad + NN Gibbs) only differ in the used models etc.

We run the gradient methods until convergence (20 000 iterations) and used the last 10 samples out of 50 to estimate each gradient for the two Gibbs sampling methods and used 100 samples for the importance sampler. For the full Gibbs sampler we drew 1000 samples from $\mathbf{A}$ of which we used the last 50 samples to estimate the mean. We initialised all algorithms using exactly the same randomly generated dictionary.

${ }^{6}$ When using a Gaussian distribution we used a variance of 3.77 , which we
estimated to fit the modified Rayleigh distribution used to generate the data. 


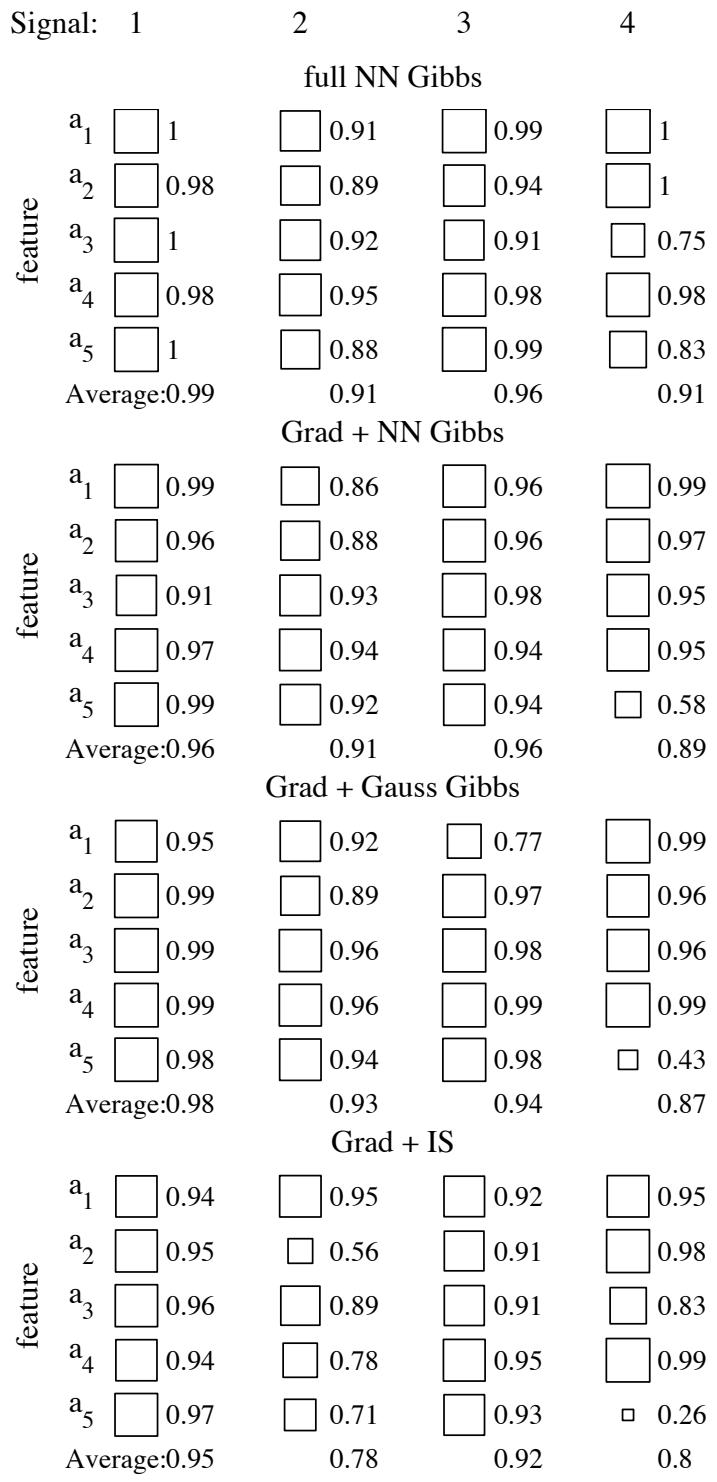

Fig. 2. Comparison of the accuracy of how well the different algorithms estimated the different features in the signal for the four different experimental setups used.

2) Results: In figure 2 we show the correlation between the normalised learned features and the closest true feature ${ }^{7}$. Figure 2 shows a Hinton style diagram in which the size of each square is proportional to the measured correlation. We also summarise the results of each column using the average over the five features.

From the above results we draw the following conclusions:

- For signal one, all algorithms show roughly similar performance;

- The importance sampler performed worse on all signals;

- Increasing the noise reduced the performance of all algorithms, in particular the importance sampler performed significantly worse for this signal;

- Decreasing the sparsity of the test signal did decrease the

\footnotetext{
${ }^{7}$ Because $\left\|\mathbf{a}_{k}\right\|_{2}=1=\left\|\widehat{\mathbf{a}_{k}}\right\|_{2}$, the correlation $\mathbf{a}_{k}^{T} \widehat{\mathbf{a}_{k}}$ is inversely proportional to the $L_{2}$ error: $\left\|\mathbf{a}_{k}-\widehat{\mathbf{a}_{k}}\right\|_{2}^{2}=2-2 \mathbf{a}_{k}^{T} \widehat{\mathbf{a}_{k}}$.
}

performance of all methods;

- Features that occurred less frequent were learnt less well, in particular the gradient methods were unable to estimate these features, while the full Gibbs sampler performed best in this task;

- Comparing the gradient methods based on the Gibbs sampler it can be observed that using the modified Rayleigh distribution did sometimes lead to worse results than using a Gaussian distribution for the non-zero coefficients;

- The non-negative prior seemed to improve learning of less frequently occurring features;

\section{Estimation of $\mathbf{s}$}

In this subsection we analyse the different sampling strategies in terms of estimation of $\mathbf{s}$. Different applications use different performance measures, for example in coding one is interested in rate-distortion, while in source separation one is interested in the distortion of the estimated sources. We therefore look at two different performance measures. First we directly compare estimates of $\mathbf{s}$ with the true $\mathbf{s}$ values used to generate the test signals. We then analyse the sparsity and reconstruction error of the approximations, i.e. the number of non-zero coefficients and the estimation error of $\mathbf{x}$.

1) Used Algorithms: To estimate s we calculated MAP estimates by drawing samples using the following three sampling strategies:

- NN: The Gibbs sampler with the modified Rayleigh distribution;

- Gauss: The Gibbs sampler with the Gaussian distribution;

- IS: The importance sampling method also with the Gaussian distribution.

We here used signal one, the exact parameters and true dictionary A (unless stated otherwise).

2) SNR in the Coefficient Domain: We show the signal to noise ratio for the estimates of $\mathbf{s}$ for the three Monte Carlo strategies in table II. It is clear from these results that the importance sampling approach does perform significantly worse in estimating $\mathbf{s}$, which should be contrasted with the performance of this method in estimating $\mathbf{A}$, which was only slightly worse when compared to the other approaches.

TABLE II

Estimation of S With the three methods assuming A And other PARAMETERS KNOWN.

\begin{tabular}{|c|c|c|c|}
\hline & NN Gibbs & G Gibbs & IS \\
\hline SNR in dB (s) & 43 & 24 & -2 \\
\hline
\end{tabular}

3) Sparsity vs. Reconstruction Error: To compare the algorithms in terms of reconstruction error and sparsity we varied the noise variance $\lambda_{\epsilon}^{-1}$ and sparsity parameter $\lambda_{u}$ to trace the three curves shown in figure 3 , where we show the results for the non-negative prior (solid line), the Gaussian prior (dashed line) and the importance sampler (dotted line). Note that we here normalised the $\mathrm{x}$-axis by dividing the estimated number of non-zero coefficients by the number of non-zero coefficients used to generate the signal. 


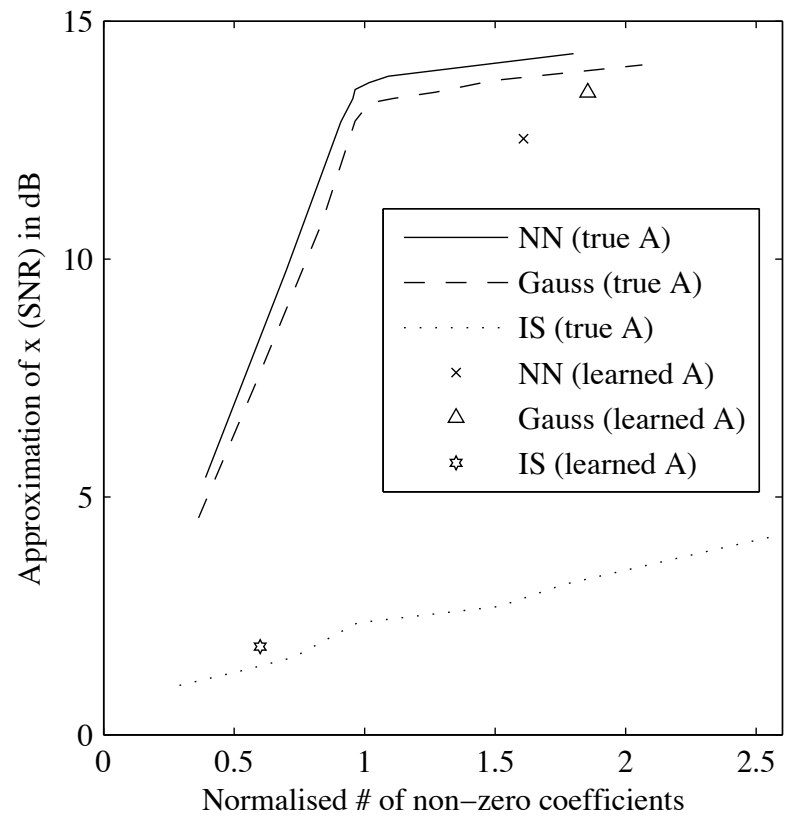

Fig. 3. The points show the reconstruction error and the number of non-zero coefficients using the MAP estimates of $\mathbf{s}$ calculated with the three sampling methods (NN, Gauss and IS) using the learned dictionaries. The lines show the results calculated with the true dictionary by varying the noise $\left(\lambda_{\epsilon}\right)$ and sparsity $\left(\lambda_{u}\right)$ in the algorithms. Solid line: NN Gibbs; dashed line: Gauss Gibbs; dotted line: IS.

In order to analyse how the estimation of $\mathbf{A}$ influences the performance in terms of reconstruction error and sparsity, we also used the learned dictionaries and the true values for $\lambda_{\epsilon}$ and $\lambda_{u}$. These results are shown as points in figure 3 .

We make the following observations:

- When using the Gibbs sampler, a better estimate of $\mathbf{A}$ leads to better approximations in terms of sparsity and reconstruction error;

- For a particular estimate of $\mathbf{A}$ we found that using the Gibbs sampler with the modified Rayleigh prior resulted in sparser and more accurate signal approximations compared to the Gibbs sampler with the Gaussian prior;

- The importance sampler does not produce good sparse approximations and the variation in the results was large.

- The best estimation of $\mathbf{s}$ (in terms of reconstruction error and sparsity) is achieved using the prior used to generate the data.

- The Gibbs sampler outperforms the importance sampler in estimating $\mathbf{s}$ when both use the same prior model.

\section{Importance Sampler Performance}

The reason behind the poor performance of the importance sampler in estimating $\mathbf{s}$ can be understood by viewing the MAP estimation as a form of random search, where the search is distributed depending on the posterior distributions. While the Gibbs sampler draws samples from the correct distributions and frequently visits areas with high probability, the importance sampler draws the samples from a different distribution and the search is less likely to search areas with high probability.

\section{COMPARISON WITH OTHER METHODS}

In this section we compare the Monte Carlo algorithms discussed in this paper and contrast the achieved results to the results obtained with several other algorithms proposed in the literature. The main focus in this paper has been on estimation of $\mathbf{A}$ and we here concentrate on this aspect.

\section{A. Test Signal}

As a test signal we use an artificial musical signal, which is motivated by our recent work on music analysis [13]. This signal was generated by using the recorded performance information of a real piano performance of Ludwig van Beethoven's Bagatelle No. 1 Opus 33 as the sparse time-series s (see [48] for more information on the data). To simplify the problem we restricted all pitches played to one octave and reduced the time scale. We then generated the signal using the 12 different features shown in figure 4, each 128 samples long and generated using an FM synthesis technique.

This signal is similar to the problem used in the previous subsection with the following exceptions; 1) the problem size is larger as it has more and longer features; 2) the individual features are highly correlated to features one semitone above or below (the correlation between adjacent features was between 0.5 and 0.7 ) and 3) the non-zero coefficients do not follow exactly the modified Rayleigh distribution. (See the histogram in figure 6 in the appendix.)

\section{B. Used Algorithms}

We here compare the following methods:

- full NN Gibbs: Sampling from $p(\mathbf{A}, \mathbf{s} \mid \mathbf{x})$ with the modified Rayleigh prior on the non-zero s;

- Grad + Gauss Gibbs: The gradient method using the Gibbs sampler with a Gaussian prior on the non-zero $\mathbf{s}$ as proposed in [22] ;

- Grad + NN Gibbs: The gradient method using the Gibbs sampler with the non-negative modified Rayleigh prior on the non-zero $\mathbf{s}$;

- Grad + IS 100 and Grad + IS 10 000: The gradient method using the importance sampler using 100 and 10000 samples $^{8}$ to estimate each gradient;

- Grad + EM: The gradient method with an estimation of the gradient based on an approximation of $p(\mathbf{s} \mid \mathbf{x}, \mathbf{A})$ with a delta function at a local maximum [8]. We used the EM algorithm of Figueiredo [18] to find the MAP estimate. This method was previously used for the sparse approximation of time-series in [13] ;

- Grad + MP: The gradient method with an estimation of the gradient based on Matching Pursuit. This method was previously used in [2];

- MoTIF: The MoTIF method proposed in [20], which is a heuristic greedy learning algorithm that forces extracted features $\mathbf{a}_{k}$ to be as dissimilar as possible.

Note that method (Grad + EM) is based on an EM algorithm and requires a different model to the one used with the Monte

\footnotetext{
${ }^{8}$ Note that increasing the number of samples reduces the bias in the gradient estimate.
} 

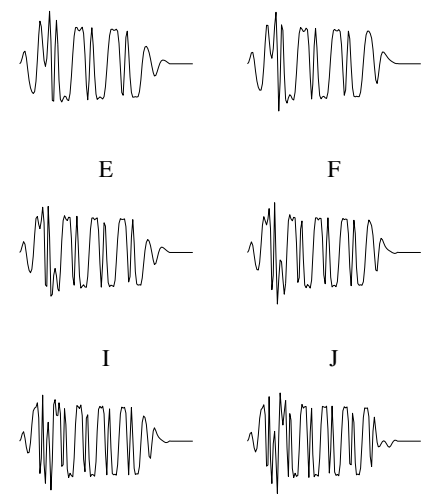

$\mathrm{C}$

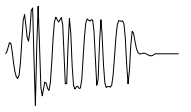

G
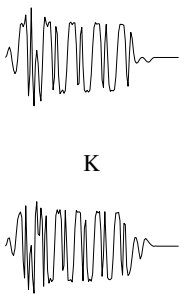

$\mathrm{D}$
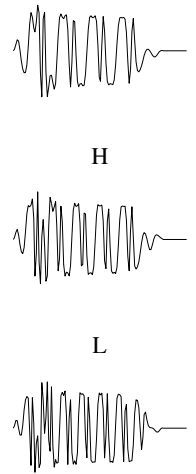

Fig. 4. The twelve features used to generate the test signal.

Carlo methods whilst the Matching Pursuit and the MoTIF algorithms are not derived from any particular probabilistic formulation.

Apart from the Matching Pursuit, the MoTIF and the importance sampling methods, we used the subset selection procedure described in [13]. This method offers a fast way to select a small number of features and their associated position based on the correlation between the observed data and the features at all shifts. Similar to thresholding methods, this method calculates the correlation between the signal and all features and then selects the subset based on this correlation alone. The difference to standard thresholding methods is that selected features are not allowed to overlap with shifts of the same feature by more than a predefined amount [13]. By using this subset selection method we restrict the sampler to explore only a subset of the distribution. As it is only possible to draw a small number of samples in practice the sampler is not able to explore the distribution sufficiently even without subset selection and we found that for a fixed computational budget the performance greatly increased when combining the sampler with the subset selection method.

\section{Initialisation}

In all experiments we initialised the features $\mathbf{a}_{k}$ with the same set of sinusoidal functions. In table III we summarise the different parameters used for the different algorithms. The first row gives the number of features to be learned, the second row (grad iter) gives the number of iterations or samples used to evaluate each gradient and the third row shows the overall number of samples drawn from $p(\mathbf{A} \mid \mathbf{x}, \mathbf{s})$ or the number of overall gradient steps (Overall iter). Numbers in parenthesis show the actual number of samples used after the burn-in period. The fourth row contains the block size used in each iteration. The fifth row shows a rough estimate of the computation time required for each algorithm.

\section{Model Parameter Estimation}

The focus of this paper was on the adaptation of the features $\mathbf{a}_{k}$. However, other model parameters can be adapted using similar methods as studied here. In [22] maximum likelihood gradient type update rules have been proposed and we present such updates for different model parameters in appendix $\mathrm{V}$. Another possible approach would be to specify priors for all model parameters and to extend the sampler to also sample from these parameters.

The results obtained below were achieved with the model parameters learned using the gradient estimates in appendix V. However, we found that the estimation performance of the $\mathbf{a}_{k}$ did not depend strongly on the other model parameters and experiments with parameters estimated from the true coefficients $\mathbf{s}$ have produced similar results.

\section{E. Results}

A quantitative analysis of the results is given in figure 5, where the correlations between the true features $\mathbf{a}_{k}$ and the closest learned features at the best shift are compared for the five methods. The figure shows a Hinton style diagram, where the correlation is related to the size of the squares shown. The last column in figure 5 shows the number of occurrences for each note.

We make the following observations:

- The Gibbs sampling methods outperform the other methods;

- The importance sampling method did produce significantly worse results for this problem than the Gibbs sampler, which we attribute to the bias;

- Combining the subset selection procedures with the Gibbs sampling methods did perform better than the fast methods;

- Again, less frequently occurring features are learned less well with all methods;

- Due to the high correlation of individual features, the features that had not been learned were often modelled quite well with features learned to represent other features and the fact that these features had not been learned did therefore not increase the overall reconstruction error significantly;

- The results obtained by sampling from $\mathbf{A}$ are slightly worse than those found with the Gibbs sampling based gradient method using the same prior. This seems to be a result of the aforementioned permutation ambiguity.

\section{CONCLUSION}

Shift-invariant sparse signal approximations are a new powerful paradigm with many promising applications to signal processing of time-series. In this paper we have studied Monte Carlo strategies to adapt the shift-invariant sparse coding model to optimally approximate a given set of observations. We found that these methods offer better performance in terms of feature learning as compared to previously suggested methods. However, Monte Carlo methods can be computationally demanding. Using a subset selection method to reduce the problem size for large problems was found to work well in practice and the combination of Gibbs sampling strategies and the subset selection method was found to outperform other methods. Nevertheless, even with the subset selection method, the algorithms can be slower than previous approaches. 
TABLE III

PARAMETERS FOR THE DIFFERENT ALGORITHMS USED IN THE EXPERIMENTS.

\begin{tabular}{|l|c|c|c|c|c|c|c|c|}
\hline Method & Full NN Gibbs & NN Gibbs & Gauss Gibbs & IS 100 & IS 10 000 & EM & MP & MoTIF \\
\hline \# of feature & 12 & 12 & 12 & 12 & 12 & 12 & 12 & 12 \\
Grad. iter & na & $50(10)$ & $50(10)$ & 100 & 10000 & 10 & na & na \\
Overall iter & $5000(500)$ & 10000 & 10000 & 10000 & 10000 & 10000 & 10000 & na \\
Block size & na & 256 & 256 & 256 & 256 & 256 & 256 & 256 \\
Time & 3 days & 3 days & 3 days & 7 hours & 5 days & 3 days & 5 minute & 1 minute \\
\hline
\end{tabular}

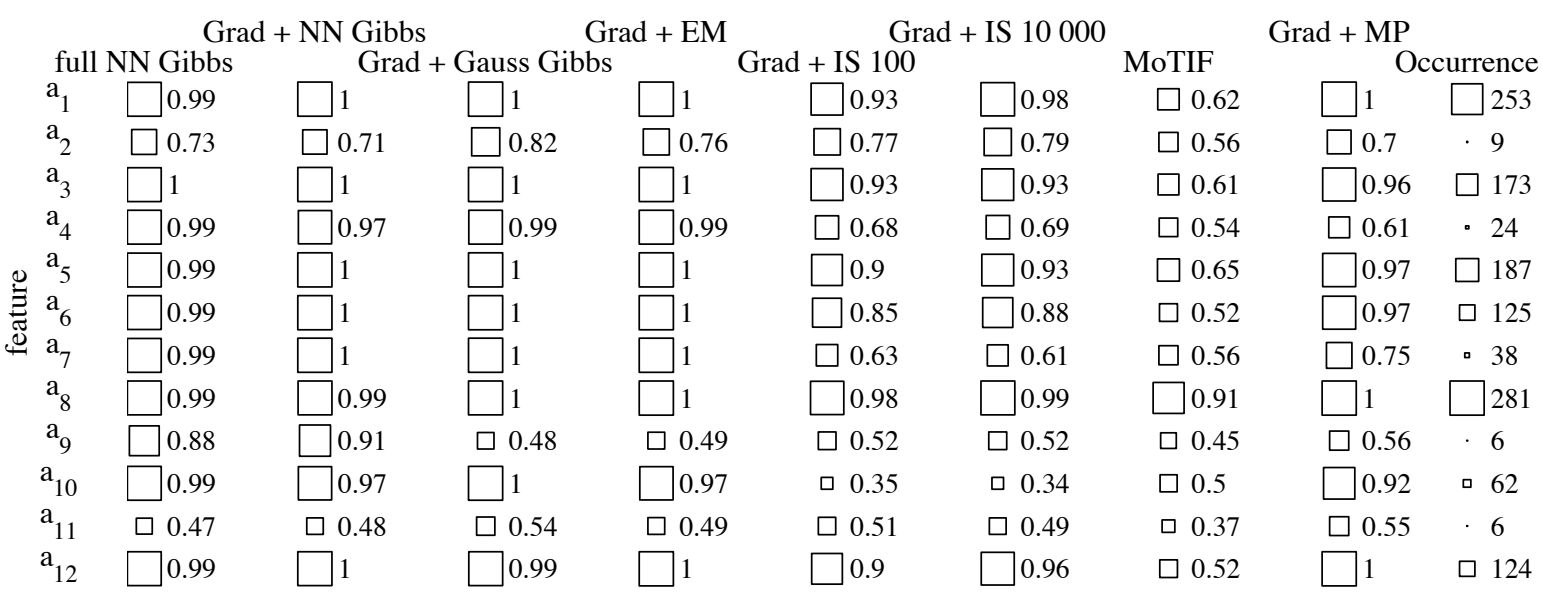

Fig. 5. Hinton style diagram showing the correlation between the learned features and the original features for the different methods. The size of the squares represents the value. The last column shows the number of occurrence of each of the features in the original signal.

The importance sampling method was faster than the Gibbs sampling based approaches. We found that for small problems this method can be used successfully to learn the features, however, for larger problems the bias introduced into the gradient estimate becomes significant and the performance was found to drop. Contrary to the performance in feature learning, the importance sampler was not able to estimate the sparse coefficients $\mathbf{s}$.

Another benefit of the sampling strategies was the ability to use a range of prior distributions. We found that choosing this distribution to closely model the data offered additional performance benefits, in particular in terms of estimation of the sparse coefficients $\mathbf{s}$ and in terms of sparsely representing a signal. The problem of estimating $\mathbf{A}$ on the other hand was found to be quite robust with respect to the used prior and the used parameters.

\section{APPENDIX I \\ DERIVATION OF EQUATION (8)}

$$
\begin{aligned}
-\frac{\partial}{\partial \mathbf{a}_{k}} \ln p(\mathbf{A} \mid \mathbf{x}) & =-\frac{\frac{\partial}{\partial \mathbf{a}_{k}} \iint p(\mathbf{A}, \mathbf{s} \mid \mathbf{x}) d \mathbf{s}}{\iint p(\mathbf{A}, \mathbf{s} \mid \mathbf{x}) d \mathbf{s}} \\
& =-\iint \frac{p(\mathbf{A}, \mathbf{s} \mid \mathbf{x})}{p(\mathbf{A} \mid \mathbf{x})} \frac{\partial}{\partial \mathbf{a}_{k}} \ln p(\mathbf{A}, \mathbf{s} \mid \mathbf{x}) d \mathbf{s} \\
& =-\iint p(\mathbf{s} \mid \mathbf{A}, \mathbf{x}) \frac{\partial}{\partial \mathbf{a}_{k}} \ln p(\mathbf{x}, \mathbf{A} \mid \mathbf{s}) d \mathbf{s}
\end{aligned}
$$

Because $\frac{\partial}{\partial \mathbf{a}_{k}} \ln p(\mathbf{A}, \mathbf{s} \mid \mathbf{x})=\frac{\partial}{\partial \mathbf{a}_{k}} \ln p(\mathbf{x}, \mathbf{A} \mid \mathbf{s})+\frac{\partial}{\partial \mathbf{a}_{k}} \ln \frac{p(\mathbf{s})}{p(\mathbf{x})}=$ $\frac{\partial}{\partial \mathbf{a}_{k}} \ln p(\mathbf{x}, \mathbf{A} \mid \mathbf{s})$.

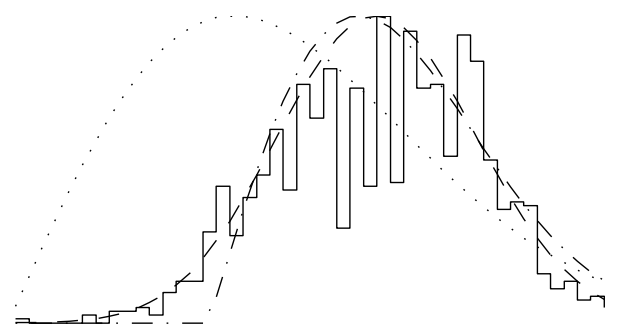

Fig. 6. A comparison between the histogram of note velocities of a real piano performance (solid) as used in section VI and the modified Rayleigh distribution introduced in the text. Also shown are a standard Rayleigh distribution (dotted) and a shifted Rayleigh distribution (dash dotted).

\section{APPENDIX II}

THE MODIFIED RAYLEIGH DISTRIBUTION

We define the modified Rayleigh distribution as:

$$
p_{m R}\left(s ; \mu, \sigma_{R}\right)= \begin{cases}\frac{1}{Z_{m R}} s e^{-(s-\mu)^{2} / 2 \sigma_{m R}^{2}} & \text { if } 0<s \\ 0 & \text { otherwise. }\end{cases}
$$

An example of this distribution is shown in figure 6 (dashed line). The normalising constant for this distribution is:

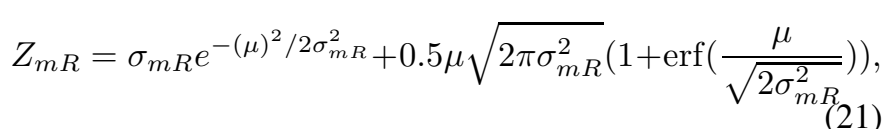

where $\operatorname{erf}(\cdot)$ is the error function.

To motivate the use of the modified Rayleigh distribution for the 'Toy' example of section VI, we also show the histogram of the note amplitudes figure 6. Also shown are the standard Rayleigh distribution with (dash dotted line) and without a shift (dotted line). 


\section{APPENDIX III}

DeRIVATION OF $E_{1}$ FOR THE GiBBS SAMPLER

A. Derivation of $E_{1}$ for $p\left(s_{n} \mid u_{n}=1\right)=p_{m R}\left(s ; \mu_{n}, \lambda_{R}^{-1}\right)$

If we use $p\left(s_{n} \mid u_{n}=1\right)=p_{m R}\left(s ; \mu_{n}, \lambda_{R}^{-1}\right)$, we have from equation (17):

$$
\begin{aligned}
E_{1}= & \log \frac{p\left(u_{n}=1\right) \int p(\mathbf{x} \mid \mathbf{s}, \mathbf{u}, \mathbf{A}) p\left(s_{n} \mid u_{n}=1\right) d s_{n}}{p\left(u_{n}=0\right) \int p(\mathbf{x} \mid \mathbf{s}, \mathbf{u}, \mathbf{A}) p\left(s_{n} \mid u_{n}=0\right) d s_{n}} \\
= & \log \frac{\int e^{-0.5 \lambda_{\epsilon}(\mathbf{x}-\mathbf{A s})^{T}(\mathbf{x}-\mathbf{A s})} \frac{1}{Z^{p}} s_{n} e^{-0.5 \lambda_{R}\left(s_{n-\mu_{n}}\right)^{2}} d s_{n}}{\int e^{-0.5 \lambda_{\epsilon}(\mathbf{x}-\mathbf{A s})^{T}(\mathbf{x}-\mathbf{A s})} \delta_{0}\left(s_{n}\right) d s_{n}} \\
& +\log e^{-0.5 \lambda_{u}}
\end{aligned}
$$

where $Z_{p}$ is the normalising constant of the modified Rayleigh distribution given in equation (21). We can write this as

$$
\begin{aligned}
E_{1}= & \log \frac{\int e^{-0.5 \lambda_{E_{n}}\left(b_{n}-s_{n}\right)^{T}\left(b_{n}-s_{n}\right)} \frac{1}{Z^{p}} s_{n} e^{-0.5 \lambda_{R}\left(s_{n-\mu_{n}}\right)^{2}} d s_{n}}{\int e^{-0.5 \lambda_{E_{n}}\left(b_{n}-s_{n}\right)^{T}\left(b_{n}-s_{n}\right)} \delta_{0}\left(s_{n}\right) d s_{n}} \\
& +\log e^{-0.5 \lambda_{u}} \\
= & \log \frac{\frac{1}{Z^{p}} \int s_{n} e^{-0.5 \Psi_{n} s_{n}^{2}+\Psi_{n} \eta_{n} s_{n}-0.5\left(\lambda_{E_{n}} b_{n 2}+\lambda_{R \mu_{n}}\right)} d s_{n}}{e^{-0.5 \lambda_{E_{n}}\left(b_{n}\right)^{2}}} \\
& +\log e^{-0.5 \lambda_{u}} \\
= & \log e^{0.5 \lambda_{E_{n}} b_{n}^{2}} e^{-0.5 \lambda_{u}} \frac{Z_{E}}{Z^{p}} \int s_{n} e^{-0.5 \Psi_{n}\left(s_{n}-\eta_{n}\right)^{2}} d s_{n},
\end{aligned}
$$

where in the last line we use

$$
Z_{E}=e^{0.5\left(\Psi_{n} \eta^{2}-\lambda_{E-n} b_{n}^{2}-\lambda_{R} \mu_{n}^{2}\right)} .
$$

The integral in the last line is the normalising constant in the modified Rayleigh distribution given in equation (21) so that the expression for $E_{1}$ in equation (16) becomes:

$$
E_{1}=-\frac{\lambda_{u}}{2}+\frac{\lambda_{E_{n}}}{2} b_{n}^{2}+\ln \Phi
$$

where

$\Phi=\frac{Z_{E}}{Z_{p}}\left[\frac{1}{\Psi_{n}} e^{-0.5 \eta^{2} \Psi_{n}}+0.5 \eta \sqrt{\frac{2 \pi}{\Psi_{n}}}\left(1+\operatorname{erf}\left(\eta \sqrt{\frac{\Psi}{2}}\right)\right)\right.$

with

$$
Z_{E}=e^{-0.5\left(-\eta^{2} \Psi_{n}+b_{n}^{2} \lambda_{E_{n}}+\mu_{n}^{2} \lambda_{R}\right)}
$$

and

$$
Z_{p}=\frac{e^{-\mu_{n}^{2} 0.5 \lambda_{R}}}{\lambda_{R}}+0.5 \mu_{n} \sqrt{\frac{2 \pi}{\lambda_{R}}}\left(1+\operatorname{erf}\left(\mu_{n} \sqrt{0.5 \lambda_{R}}\right)\right) .
$$

$\eta_{n}$ and $\frac{1}{\Psi_{n}}$ are the parameters of the posterior $p\left(s_{n} \mid s_{\hat{n} \neq n}, u_{n}=1, \mathbf{A}\right)$, which due to the conjugate prior is also of the modified Rayleigh form. The parameters are given analytically as:

$$
\eta_{n}=\frac{\lambda_{E_{n}} b_{n}+\lambda_{R} \mu_{n}}{\lambda_{E_{n}}+\lambda_{R}}
$$

and

$$
\Psi_{n}=\lambda_{E_{n}}+\lambda_{R}
$$

Here we have used the notation $\lambda_{E_{n}}=\lambda_{\epsilon} \mathbf{a}_{n}^{T} \mathbf{a}_{n}$ and $b_{n}=$ $\left(\mathbf{a}_{n}^{T} \mathbf{a}_{n}\right)^{-1} \mathbf{a}_{n}^{T} \mathbf{x}$.

\section{B. Derivation of $E_{1}$ for $p\left(s_{n} \mid u_{n}=1\right) \sim \mathcal{N}\left(s ; 0, \lambda_{R}^{-1}\right)$}

In [35] the derivation of $E_{1}$ is presented for the case in which $p\left(s_{n} \mid u_{n}=1\right)=\mathcal{N}\left(s ; 0, \lambda_{R}^{-1}\right)$. Using the notation introduced above for $\Psi_{n}$ and using $\mu_{n}=0$ we get:

$$
E_{1}=-\frac{\lambda_{u}}{2}+\frac{\lambda_{E_{n}}}{2} b_{n}^{2}+0.5 \ln \frac{2 \pi}{\Psi_{n}}-0.5 \lambda_{E_{n}} b_{n}^{2}\left(1+\frac{\lambda_{E_{n}}}{\Psi_{n}}\right) .
$$

\section{APPENDIX IV}

\section{SAMPLING FROM THE MODIFIED RAYLEIGH DISTRIBUTION}

The modified Rayleigh distribution can be written as:

$$
\begin{aligned}
& \frac{1}{Z_{m R}} s e^{-(s-\mu)^{2} / 2 \sigma_{m R}^{2}}= \\
& \quad \frac{1}{Z_{m R}}\left((s-\mu) e^{-(s-\mu)^{2} / 2 \sigma_{m R}}+\mu e^{-(s-\mu)^{2} / 2 \sigma_{m R}}\right) .
\end{aligned}
$$

This form suggests a hybrid sampling strategy. With probability $\frac{1}{Z_{m R}}\left(\sigma_{m R}+0.5 \mu \sqrt{2 \pi \sigma_{m R}}\right), s>\mu$ and we can sample from:

$$
\begin{aligned}
p(s \mid s>\mu) & =\mu+\left[\frac{\sigma_{m R}}{Z_{m R}}\right] \sigma_{m R}^{-1} s e^{-0.5 \sigma_{m R}^{-1} s^{2}} \\
& +\left[0.5 \frac{\mu}{Z_{m R}} \sqrt{2 \pi \sigma_{m R}}\right] 2 \sqrt{\frac{\sigma_{m R}^{-1}}{2 \pi}} e^{-0.5 \sigma_{m R}^{-1} s^{2}},
\end{aligned}
$$

which is a mixture of a truncated Gaussian and a shifted Rayleigh distribution.

For $s<\mu$ we have an upper bound on the distribution of

$$
\frac{1}{Z_{m R}} \mu e^{-(s-\mu)^{2} / 2 \sigma_{m R}}
$$

in which case rejection sampling can be used.

\section{APPENDIX V}

\section{GRADIENT EXPRESSIONS FOR THE OTHER MODEL}

\section{PARAMETERS}

Gradient expressions for other model parameters (say $\theta$ ) can be derived in a similar way to those in equation (8).

$$
\Delta \theta=\frac{\partial \log p(\mathbf{A} \mid \mathbf{x})}{\partial \theta}=\left\langle\frac{\partial}{\partial \theta} \log p(\mathbf{x}, \mathbf{A} \mid \mathbf{s})\right\rangle .
$$

Again we assume that the prior for the model parameters is relatively flat, so that the gradient of this prior is set to zero. We then get the gradient for the noise scale factor $\lambda_{\epsilon}$

$$
\Delta \lambda_{\epsilon}=\left\langle\frac{M}{2 \lambda_{\epsilon}}-\frac{1}{2}(\mathbf{x}-\mathbf{A} \mathbf{s})^{T}(\mathbf{x}-\mathbf{A} \mathbf{s})\right\rangle,
$$

and the gradient of the parameter $\lambda_{u}$ in the Bernoulli prior:

$$
\Delta \lambda_{u}=\left\langle\frac{N}{2\left(1+e^{\frac{\lambda_{u}}{2}}\right)}-\frac{1}{2} \mathbf{u}^{T} \mathbf{u}\right\rangle .
$$

For the model using a Gaussian prior for the non-zero coefficients $s$ we get a gradient for the scale factor for the Gaussian $\lambda_{R}$

$$
\Delta \lambda_{R}=\left\langle\frac{\mathbf{u}^{T} \mathbf{u}}{2 \lambda_{G}}-\frac{1}{2} \mathbf{s}^{T} \mathbf{s}\right\rangle
$$


while for the model in which the non-zero coefficients $\mathrm{s}$ have a modified Rayleigh prior we get a gradient for the modified Rayleigh parameter $\lambda_{R}$

$$
\Delta \lambda_{R}=\left\langle-0.5 \sum_{s_{j n} \neq 0}\left(s_{j n}-\mu\right)^{2}-\frac{U}{c_{1}}\left(-\frac{0.5 \mu c_{2}}{\lambda_{R}}-c_{3} \lambda_{R}^{-2}\right)\right\rangle
$$

and for the modified Rayleigh parameter $\mu$

$$
\Delta \mu=\left\langle\sum_{s_{j n} \neq 0} \lambda_{R}\left(s_{j n}-\mu\right)-\frac{U}{c_{1}} c_{3}\right\rangle .
$$

Here $\mathrm{U}$ is the number of the non-zero $s, c_{1}=\mu c_{2}+\lambda_{R}^{-1} c_{3}$, $c_{2}=0.5 \sqrt{2 \pi \lambda_{R}^{-1}}\left(1+\operatorname{erf}\left(\mu \sqrt{0.5 \lambda_{R}}\right)\right)$ and $c_{3}=e^{-0.5 \lambda_{R} \mu^{2}}$. We also use $\langle\cdot\rangle$ to denote the expectation with respect to $p(\mathbf{s}, \mathbf{u} \mid \mathbf{x}, \mathbf{A})$, which can again be approximated using Monte Carlo estimates.

\section{ACKNOWLEDGMENT}

The authors would like to thank the anonymous reviewers for additional references and valuable comments, which greatly helped to improve this article.

\section{REFERENCES}

[1] V. K. Goyal, M. Vetterli, and N. T. Thao, "Quantized overcomplete expansions in $R^{N}$ : Analysis, synthesis and algorithms," IEEE Transactions on Information Theory, vol. 44, pp. 16-31, Jan. 1998.

[2] E. C. Smith and M. S. Lewicki, "Efficient auditory coding," Nature, vol. 439, pp. 978-982, Feb. 2006.

[3] R. Figueras i Ventura, P. Vandergheynst, and P. Frossard, "Low rate and flexible image coding with redundant representations," to appear in IEEE Transactions on Image Processing.

[4] M. E. Tipping, "Sparse Bayesian learning and the relevance vector machine," Journal of Machine Learning Research, vol. 1, pp. 211-244, 2001.

[5] M. Zibulevsky and B. A. Pearlmutter, "Blind source separation by sparse decomposition in a signal dictionary," Neural Computation, vol. 13, no. 4, pp. 863-882, 2001.

[6] M. Zibulevsky and P. Bofill, "Underdetermined blind source separation using sparse representations," Signal Processing, vol. 81, no. 11, pp. 2353-2362, 2001.

[7] A. Hyvärinen, "Sparse code shrinkage: Denoising of nongaussian data by maximum likelihood estimation," Neural Computations, vol. 11, no. 7 , pp. 1739-1768, 1998.

[8] B. A. Olshausen and D. J. Field, "Emergence of simple-cell receptive field properties by learning a sparse code for natural images," Nature, vol. 13, pp. 607-609, Jun 1996.

[9] M. S. Lewicki and B. A. Olshausen, "Infering sparse, overcomplete image codes using an efficient coding framework," in Advances in Neural Information Processing Systems (NIPS), vol. 10, 1998.

[10] A. Holobar and D. Zazula, "Correlation-based decomposition of surface electromyograms at low contraction forces," Medical and Biological Engineering and Computing, vol. 42, pp. 487-495, 2004.

[11] T. Blumensath and M. Davies, "Shift-invariant sparse coding for single channel blind source separation," in Proceedings of the Workshop on Signal Processing with Adaptative Sparse Structured Representations, (Rennes, France), Nov. 2005.

[12] S. Abdallah, Towards Music Perception by Redundancy Reduction and Unsupervised Learning in Probabilistic Models. PhD thesis, King's College London, February 2003.

[13] T. Blumensath and M. Davies, "Sparse and shift-invariant representations of music," IEEE Transactions on Audio, Speech and Language Processing, vol. 14, pp. 50-57, Jan 2006.

[14] M. Plumbley, S. Abdallah, T. Blumensath, and M. Davies, "Sparse representations of polyphonic music," ELSVIR Signal Processing, vol. 86, pp. 417-431, March 2006.

[15] G. Davis, Adaptive Nonlinear Approximations. PhD thesis, New York University, 1994.
[16] S. Mallat and Z. Zhang, "Matching pursuits with time-frequency dictionaries," IEEE Transactions on Signal Processing, vol. 41, no. 12, pp. 3397-3415, 1993.

[17] S. S. Chen, D. L. Donoho, and M. A. Saunders, "Atomic decomposition by basis pursuit," SIAM Journal of Scientific Computing, vol. 20, no. 1, pp. 33-61, 1998.

[18] M. A. T. Figueiredo, "Adaptive sparseness for supervised learning," IEEE Transactions on Pattern Analysis and Machine Intelligence, vol. 25, pp. 1150-1159, Sept. 2003.

[19] K. Kreutz-Delgado, J. F. Murray, B. D. Rao, K. Engan, T.-W. Lee, and T. J. Sejnowski, "Dictionary learning algorithms for sparse representation," Neural Computation, vol. 15, no. 2, pp. 349-396, 2003.

[20] P. Jost, P. Vandergheynst, S. Lesagne, and R. Gribonval, "MoTIF: An efficient algorithm for learning translation invariant dictionaries," in Proc. of the Int. Conf. on Accoustic, Speech and Signal Processing, May 2006.

[21] B. A. Olshausen and K. Millman, "Learning sparse codes with a mixtureof-gaussians prior," in Advances in Neural Information Processing Systems (NIPS), pp. 841-847, 2000.

[22] P. Sallee and B. A. Olshausen, "Learning sparse multiscale image representations," in Advances in Neural Information Processing Systems (NIPS), pp. 1327-1334, 2003.

[23] T. Blumensath and M. Davies, "Enforcing sparsity, shift-invariance and positivity in a Bayesian model of polyphonic music," in Proc. of the IEEE Workshop on Statistical Signal Processing, July 2005.

[24] T. Blumensath and M. Davies, "A fast importance sampling algorithm for unsupervised learning of over-complete dictionaries," in Proc. of the Int. Conf. on Acoustics, Speech and Signal Processing, pp. 213-216, March 2005.

[25] M. S. Lewicki and T. J. Sejnowski, "Coding time-varying signals using sparse, shift-invariant representations," in Advances in Neural Information Processing Systems (NIPS), vol. 11, pp. 730-736, 1999.

[26] R. Bogacz, M. W. Brown, and C. Giraud-Carrier, "Emergence of movement-sensitive neurons' properties by learning a sparse code of natural moving images," in Advances in Neural Information Processing Systems (NIPS), pp. 838-844, 1999.

[27] B. A. Olshausen, "Sparse coding of time-varying natural images," in Proc. of the Int. Conf. on Independent Component Analysis and Blind Source Separation, 2000.

[28] H. Wersing, J. Eggert, and E. Körner, "Sparse coding with invariance constraints," in Proc. Int. Conf. Artificial Neural Networks ICANN, pp. 385-392, 2003.

[29] E. Smith and M. S. Lewicki, "Efficient coding of time-relative structure using spikes," Neural Computation, vol. 17, pp. 19-45, Jan 2005.

[30] C. P. Robert and G. Casella, Monte Carlo Statistical Methods. Springer Texts in Statistics, Springer-Verlag, 1999.

[31] A. Hyvärinen, J. Karhunen, and E. Oja, Independent Component Analysis. John Wiley \& Sons Inc, 2001.

[32] M. Girolami, "A variational method for learning sparse and overcomplete representations," Neural Computaion, vol. 11, pp. 2517 - 2532, 2001.

[33] C. Fevotte and S. Godsill, "A bayesian approach for blind separation of sparse sources," IEEE Transactions on Speech and Audio Processing, vol. PP, no. 99, pp. 1-15, 2005.

[34] M. S. Lewicki and T. J. Sejnowski, "Learning overcomplete representations," Neural Computation, no. 12, pp. 337-365, 2000.

[35] J. Geweke, "Variable selection and model comparison in regression," in Bayesian Statistics 5. (J. M. Bernardo, J. O. Berger, A. P. Dawid, and A. F. M. Smith, eds.), Oxford University Press, 1996.

[36] K. Kreutzer-Delgado, B. D. Rao, and K. Engan, "Convex/ Schur-Convex (CSC) log-priors and sparse coding," in Proc. of the 6th Joint Symposium on Neural Computation, (Pasadena, California), May 1999.

[37] H. J. Kushner and G. G. Yin, Stochastic Approximation and Recursive Algorithms and Applications. Springer-Verlag New York, 2003.

[38] G. Celeux, M. Hurn, and C. P. Robert, "Computational and inferential difficulties with mixture posterior distributions," J. American Statist. Assoc., vol. 9, no. 3, pp. 957-979, 2000.

[39] M. Stephens, "Dealing with label-switching in mixture models," Journal of the Royal Statistical Society, Series B, vol. 62, pp. 795-809, 2000.

[40] M. S. Lewicki and B. A. Olshausen, "A probabilistic framework for the adaptation and comparison of image codes," J. Opt. Soc. Am. A: Optics, Image Science, and Vision, vol. 16, no. 7, pp. 1587-1601, 1999.

[41] M. Smith and R. Kohn, "Nonparametric regression using Bayesian variable selection," Journal of Econometrics, vol. 75, no. 2, pp. 317-343, 1996.

[42] J. Diebolt and C. P. Robert, "Estimation of finite mixture distributions through Bayesian sampling," Journal of the Royal Statistical Society. Series B, vol. 56, no. 2, pp. 363-375, 1994. 
[43] R. E. McCulloch and E. I. George, "Approaches for Bayesian variable selection,” Statistica Sinica, vol. 7, no. 2, pp. 339-374, 1997.

[44] C. Han and B. P. Carlin, "Markov chain Monte Carlo methods for computing Bayes factors: A comparative review," Journal of the American Statistical Association, vol. 96, no. 455, pp. 1122-1134, 2001.

[45] C. Andrieu, P. Djuric, and A. Doucet, "Model selection by MCMC computation," Signal Processing, Special Issue on MCMC for Signal Processing, vol. 81, no. 1, pp. 19-37, 2001.

[46] C. Févotte and S. J. Godsill, "Sparse linear regression in unions of bases via Bayesian variable selection," IEEE Signal Processing Letters, vol. 13, pp. 441-444, Jul. 2006.

[47] R. E. McCulloch and E. I. George, "Variable selection via Gibbs sampling," Journal of the American Statistical Association, pp. 881889., September 1993.

[48] J. P. Bello, Towards the Automated Analysis of Simple Polyphonic Music: A Knowledge-based Approach. PhD thesis, Queen Mary, University of London, 2003.

\begin{tabular}{|l|} 
\\
\\
PLACE \\
PHOTO \\
HERE \\
\end{tabular}

Thomas Blumensath received the BSc hons. degree in music technology from Derby University, Derby, U.K., in 2002 and his Ph.D. degree in electronic engineering from Queen Mary, University of London, U.K., in 2006. He is currently a postdoctoral research fellow in the Institute for Digital Communication at the University of Edinburgh, working on sparse representations for signal processing and coding. His research interests include mathematical and Bayesian methods in signal processing, unsuapplications to music and audio analysis. pervised learning, sparse signal approximations and

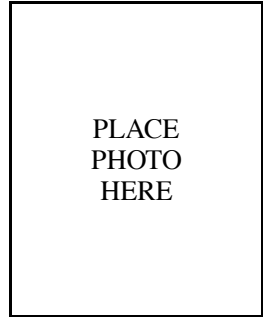

Mavies received a B.A. hons, degree in engineering from Cambridge University, Cambridge, U.K., in 1989 and the Ph.D. degree in nonlinear dynamics and signal processing from University College, London, U.K., in 1993. He was then awarded a prestigious Royal Society Research Fellowship which he held at UCL and then Cambridge. He is currently a Professor in the Institute for Digital Communications, at the University of Edinburgh and an Associate Editor for IEEE Transactions in Speech, Language and Audio Processing. His research interests include: statistical signal processing, audio processing, array signal processing, and information theory. 